\title{
3D In-Vivo Measurement of Skin Topography Using Photometric Stereo
}

\author{
Ali SOHAIB ${ }^{* a}$, Abdul FAROOQ ${ }^{a}$, Lyndon SMITH ${ }^{a}$, Melvyn SMITH ${ }^{a}$, Robert WARR ${ }^{b}$ \\ ${ }^{a}$ Machine Vision Lab, University of the West of England Bristol, UK; \\ ${ }^{\mathrm{b}}$ Department of Plastic Surgery, North Bristol NHS Trust, Bristol, UK
}

\begin{abstract}
Increased demand for treatment of skin wrinkles has led to a proliferation of different pharmaceutical and surgical treatment methods. However there still remains a lack of quantitative, objective, easily utilizable and reproducible wrinkle classification system by which these treatments can be studied and compared. We propose the use of a novel photometric stereo based technique as a potentially efficient and economical way for quantitative evaluation of skin topography. Also presented is an algorithm for improving depth estimates of skin using photometric stereo and Fractals based technique for quantification of skin topography which unlike existing $2 \mathrm{D}$ roughness parameters makes use of complete 3D topographical information.
\end{abstract}

Keywords: Photometric stereo, skin microrelief, reflectance, fractals

\section{Introduction}

\subsection{Existing wrinkle grading systems and imaging techniques}

In the absence of practical and effective 2D or 3D skin evaluation techniques, clinicians make use of wrinkle grading scales for assessing the efficacy of skin rejuvenation techniques, photo damage analysis and skin ageing. These grading scales can be categorised into three types i.e. descriptive, visual analogue and photographic scales. Most of these scales are intended for assessment of specific body regions, such as cheek sagging, crow's feet [1], nasolabial folds [2], lip fullness [3], marionette lines [4], forehead wrinkles [5] while a few are intended for the entire face [6]. The drawback of these scales is that the quality of scoring is strongly influenced by the level of experience of the examiner as the assessment is based on a subjective visual perception of lines and wrinkles rather than actual physical measurements. The scales are not malleable to different ethnic groups and suffer from low inter-observer agreement and intra-observer repeatability [7].

Several 2D and 3D techniques do exist that are intended to offer a more objective analysis of wrinkle treatments. These include line scanning based techniques, that are used for indirect measurement of skin relief, as well as mechanical and optical microscopy amongst these. However, their low scanning speed makes them unsuitable for everyday clinical analysis. Light transmission [8] is another indirect technique that uses replicas of skin for analysis, however this type of technique is very sensitive to artifacts in the replica moulds. A special vacuum pump for mixing replica material is required and this can be expensive and time consuming. Laser triangulation and fringe projection based techniques have also been used for in-vivo 3D skin surface analysis [9], [10], however they are limited to small area measurements and are also costly.

A Photometric stereo based approach requires a single camera and multiple light sources for acquisition. It provides faster acquisition and processing times (depending on the type of camera used) and is cost effective. However its applications involving in-vivo quantitative analysis of skin roughness and topography measurement over larger areas, such as regions of the face, are scarce as the complex optical mechanism of the skin makes it difficult to obtain accurate recovery of the 3D skin topography. This paper presents a method to overcome these limitations and validates the skin measurements produced by this novel implementation of photometric stereo.

\subsection{Interreflections in skin}

The assumption associated with photometric stereo - that light incident on a surface is reflected from a single point - does not apply to concave shapes as they tend to cause light to bounce multiple times before returning to the detector, a phenomenon known as interreflection or mutual illumination. Wrinkles in the skin also suffer from interreflections as they are concave shapes, however the problem is different for skin from most materials as skin is not homogeneous or opaque and has complex optical properties that are not only different for various ethnic groups but also for area of the body under inspection. The published literature suggests that complex surfaces that exhibit interreflections

\footnotetext{
* Corresponding author: ali2.sohaib@live.uwe.ac.uk; http://www1.uwe.ac.uk/et/mvl/people.aspx
} 
have limited the application of photometric stereo for recovering $3 \mathrm{D}$ texture and $2 \mathrm{D}$ albedo. Yet it is highly suited to types of surfaces that contain fine texture, such as skin. This paper addresses this issue head on and proposes and describes an implementation of a novel methodology to mitigate the effects of interreflection when imaging human skin.

Interreflections in general are dependent on surface albedo, higher the albedo higher will be the interreflections while low albedo surfaces suffer less from interreflections as investigated by Forsyth [11]. As a result of these interreflections, photometric stereo underestimates the depth at concave regions of the surface and gives a shallower shape of the original. Most of the work regarding interreflections has been done on opaque lambertian materials with the most relevant work done regarding interreflections in skin being based on the analysis of skin replicas [12], [13]. The analysis based on skin replicas is different from actual skin as the optics and BRDF (Bidirectional reflectance distribution function) is different for both materials. Unlike replicas, human skin is translucent and has complex reflectance properties which not only vary with wavelength but also vary for different skin types (ethnicities).

\section{Skin Optics}

In trying to objectively analyse wrinkles, we are interested in the shape of the skin at the surface- its micro topography. Imaging this in any form has to account for the complex interactions of light with the skin. The outer layers of skin are not opaque. What we see is a composition of the effect that both the micro topography of the surface as well as its underlying constituents have. Inherently skin can absorb and scatter light (part of the later leading to the measured reflectance). Over the entire visible spectrum, and for each skin type, the red part of the spectrum has the highest reflectance as shown in Fig 1. This decreases as the wavelength of light decreases towards the blue part of the spectrum. This variation in reflectance as a function of wavelength is very important, as the inter-reflection phenomenon is closely related to the surface albedo/reflectance.

Areas such as grooves/wrinkles in the skin are most susceptible to inter-reflections as the hollow rounded/v-shaped geometry force light to bounce multiple times before reaching the camera sensor. However at shorter wavelengths the skin absorbs more light which increases the chances of absorption of a photon after inter-reflection, unlike at longer wavelengths, where the chances of photons reaching the camera sensor are greater as the skin absorption is less.

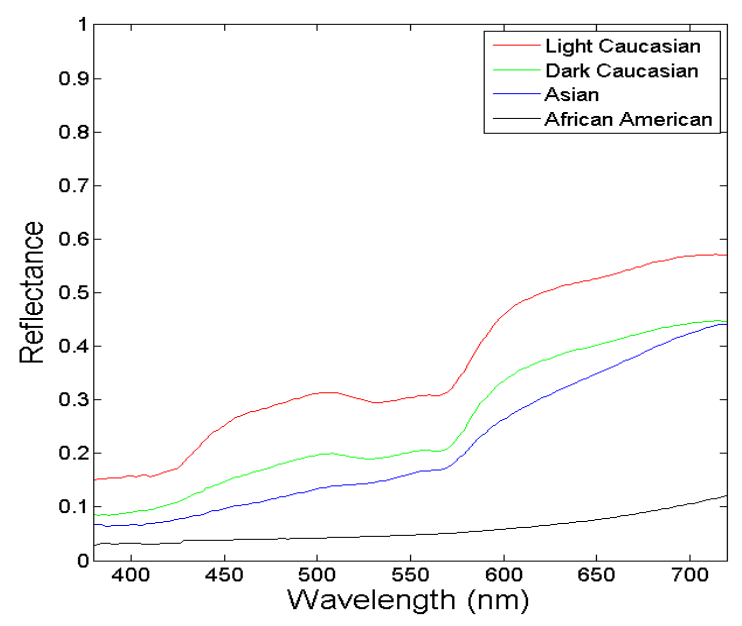

Fig. 1: Multispectral reflectance of different skin types (NCSU skin reflectance data [14]).

Also important is the variation of skin BRDF with wavelength. BRDF describes how light is reflected from a surface and is represented as.

$$
\operatorname{BRDF}\left(\theta_{\mathrm{i}}, \phi_{\mathrm{i}}, \theta_{0}, \phi_{0}, \mathrm{x}, \mathrm{y}, \lambda\right)
$$

It is a function of incoming and outgoing light direction in spherical coordinates $\left(\theta_{\mathrm{x}}\right.$ and $\phi_{\mathrm{x}}$, spatial position ( $x$ and $y)$ and wavelength $(\lambda)$. Its accurate description for a material under inspection can improve the accuracy of shape from shading algorithms, such as photometric stereo, which in its basic form assumes Lambertian BRDF. Most of the reported BRDF/BTF (Bidirectional Texture Function) measurements done for skin represent aggregates over the visible spectrum [15-17] and ignore variation in BRDF at individual wavelengths. However the measurements undertaken by [18] were 
over $544 \mathrm{~nm}$ and $633 \mathrm{~nm}$ wavelengths using a $C A S I \circledast$ scatterometer device. These measurements show that there is considerable variation in skin BRDF for these wavelengths (i.e. green and red part of the visible spectrum) and is significantly different from Lambertian BRDF.

\section{Methodology}

\subsection{Photometric stereo}

Photometric stereo uses multiple images obtained from the same viewpoint but under different directions of illumination to recover surface normal data which in turn are used to determine the 3D surface topography. The photometric stereo technique implemented is based on the Lambertian reflectance model to extract the normal vector of every point on the image plane. According to Lambertian reflectance model, the reflected intensity at a point $(i, j)$ on a surface with orientation $n=$ $\left(n_{x}, n_{y}, n_{z}\right)$, illuminated from light direction $s=[\cos \theta \sin \sigma, \sin \theta \cos \sigma, \cos \sigma](\theta$ and $\sigma$ are the tilt and slant angle respectively) is represented by:

$$
I=\rho k \cos \theta_{i}
$$

Where $p$ is the albedo, $k$ is the light source intensity and $\theta_{\mathrm{i}}$ is the angle between unit light and normal vector. For 4 light sources, the image irradiance from each light direction is given by:

$$
\begin{aligned}
& I_{1}=\rho n \cdot s_{1} \\
& I_{2}=\rho n \cdot s_{2} \\
& I_{3}=\rho n . s_{3} \\
& I_{4}=\rho n \cdot s_{4}
\end{aligned}
$$

This can be extended to $m$ light sources

$$
I_{m}=\rho n \cdot s_{m}
$$

Using (7) the surface normal can be calculated using the linear least square method.

$$
n=\left(s^{T} s\right)^{-1} s^{T} I
$$

Our photometric stereo device consists of 4 high power colour LEDs LZC-A0MD40 and a AVT Pike F100C camera. The dominant wavelength for each colour light is $462 \mathrm{~nm}, 523 \mathrm{~nm}$ and $625 \mathrm{~nm}$. The acquisition speed is around $0.6 \mathrm{~s}$ and the measurement area is $65 \times 65 \mathrm{~mm}^{2}$ approx. The device has a $10.6 \mathrm{~mm}$ depth of field to recover both micro and macro topography of skin as shown in Fig. 2.

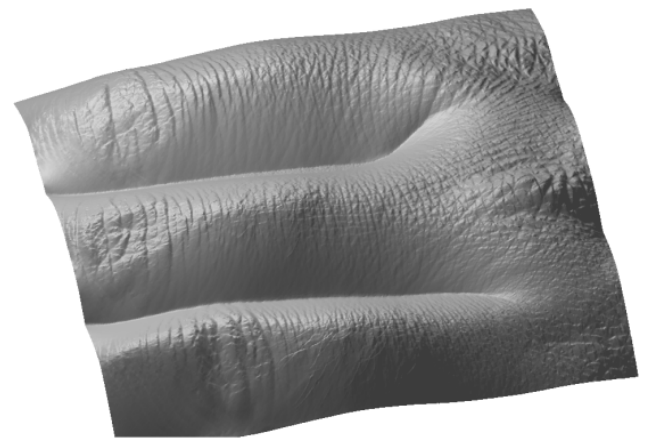

Fig. 2. 3D skin topography acquired using our photometric stereo device.

\subsection{Skin replica and ground truth}

To validate the measurements produced by our system four subjects were selected; among them, two were Caucasians and the other two Asians. The measurements were done on the forehead of all subjects by first imaging them directly using our photometric stereo device and then by producing a corresponding replica for the same skin location for each subject. All replicas were made using the SILFLO $\otimes$ impression material. The material has excellent flow and hardening characteristics, can reproduce very fine skin texture and has been widely used before for very fine replication of skin features [19-21]. These replicas were then imaged using a PRIMOS $4 \otimes$ device and used as a ground truth. The PRIMOS device is based on the principle of structured light 3D imaging and has already proven to work for wrinkles and scar evaluation and efficacy testing of skin treatments [10], [22-24]. 


\section{Error Analysis}

A cross-sectional view of the wrinkled region obtained from our photometric stereo based device is shown in Fig. 3. It is clear that at concave parts of the skin (wrinkles) the error is significant for each colour light as red light suffered the most from interreflections due to lower albedo and underestimated the wrinkle depth while green and blue light overestimated the wrinkle depth due to non-diffuse BRDF. Since white light is an aggregate of all three colours it also overestimated the wrinkle as it has one part of red and two parts of green and blue combined.

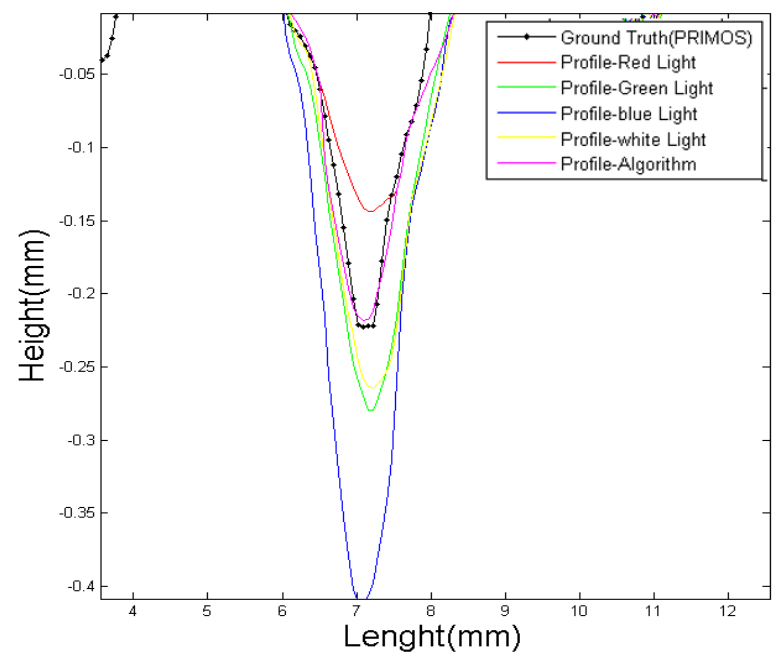

Fig. 3. Cross-sectional view of the wrinkled region obtained from each color light and our proposed algorithm.

However these errors in depth estimates were not only because of the integration of surface normals, there was exaggeration in slope from green and blue light while the red light underestimated the actual slope. Table 1 shows the root mean square (RMS) error in height for all subjects and for each individual colour light. The RMS error in height shows that the error is minimum while using red light for all subjects and it increases as the wavelength decreases from green towards blue light. Table 1 and 2 also show the improvement in depth estimates and surface normal after application of our algorithm which is explained in section 5 .

Table 1. RMS error in height for each subject and the corresponding light used (subject 2, 3 were Asians and 1,4 Caucasians).

\begin{tabular}{|l|c|c|c|c|c|}
\hline & \multicolumn{5}{|c|}{ RMS Error } \\
& Red & Green & \multicolumn{1}{c|}{ Blue } & \multicolumn{1}{c|}{ White } & Algorithm \\
\hline Subject 1 & 3.0967 & 3.6121 & 5.1534 & 3.5361 & 2.0581 \\
\hline Subject 2 & 1.1629 & 1.0340 & 2.1006 & 1.2643 & 0.8265 \\
\hline Subject 3 & 0.8796 & 1.0987 & 2.501 & 1.1161 & 0.3218 \\
\hline Subject 4 & 1.2343 & 2.3735 & 3.9193 & 1.9255 & 0.9172 \\
\hline
\end{tabular}

Table 2. 12-Norm error for all subjects from each light and proposed method (subject 2, 3 were Asians and 1,4 Caucasians).

\begin{tabular}{|l|c|c|c|c|c|}
\hline & \multicolumn{5}{|c|}{ L2-Norm Error } \\
& Red & Green & \multicolumn{1}{c|}{ Blue } & \multicolumn{1}{c|}{ White } & Algorithm \\
\hline Subject 1 & 0.2442 & 0.3660 & 0.404 & 0.3471 & 0.2168 \\
\hline Subject 2 & 0.1823 & 0.2264 & 0.2929 & 0.2294 & 0.1766 \\
\hline Subject 3 & 0.2291 & 0.2986 & 0.3322 & 0.3164 & 0.1668 \\
\hline Subject 4 & 0.2802 & 0.3352 & 0.3844 & 0.3320 & 0.2711 \\
\hline
\end{tabular}




\section{Algorithm for improved depth estimate}

Since topographic skin recovery error is largely due to interreflection in the wrinkled part of the skin, segmentation of the region of interest $(\mathrm{ROI})$ is necessary before application of the new algorithm. Here this is done by using the watershed algorithm. The accuracy of the watershed algorithm in segmenting the ROI was found to be much better when the height map of the surface was used instead of a more conventional 2D image, which has the tendency to suffer from over segmentation [25]. The surface height map was calculated using a photometric stereo algorithm and was filtered using a 3D Gaussian filter to separate high frequency data from the underlying form. The 3D Gaussian filter function used was simply a product of two Gaussian filters [26].

$$
\mathrm{S}(x, y)=\frac{1}{\mathrm{a}^{2} \cdot \lambda \mathrm{xc} \cdot \lambda \mathrm{yc}} \exp \left[-\left[\pi\left(\frac{x}{\alpha \cdot \lambda x c}\right)^{2}+\pi\left(\frac{y}{\alpha . \lambda y c}\right)\right]^{2}\right]
$$

The high frequency data was then fed into the watershed algorithm to segment the wrinkle. Fig. 4 shows the result of a segmented wrinkle for one of the subjects.

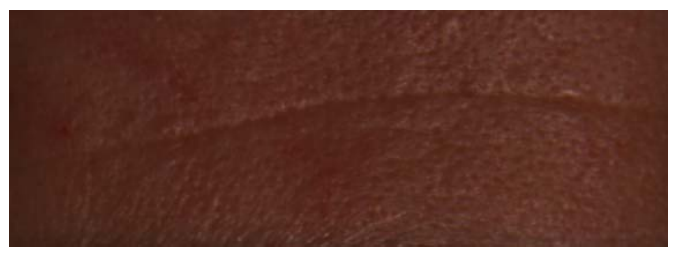

(a)

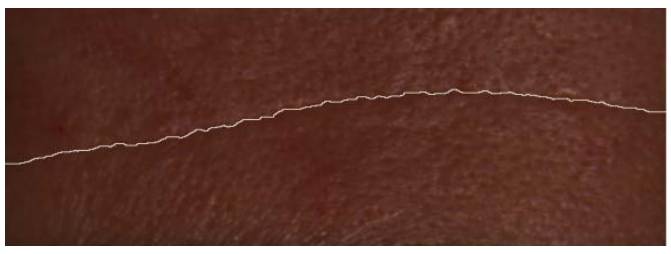

(b)

Fig. 4: (a) original image. (b) Segmented wrinkle line using watershed algorithm.

The algorithm works by scaling gradients at wrinkled regions. The segmented line from the watershed algorithm provides the points where the gradients need to be scaled. The gradients from red light were used for the whole surface and then the algorithm modifies the gradient map by adding scaled gradients at the wrinkled region only. This modified gradient map is used to calculate interim height map using the Frankot Chellappa's technique. This interim height map has values close to ground truth at the wrinkled region only. These values are substituted in the height map obtained from red light; this way the overall surface stays undisturbed except at the wrinkled region where there was underestimation of depth due to interreflection. The following pseudo code explains the steps involved in improvement of depth estimates.

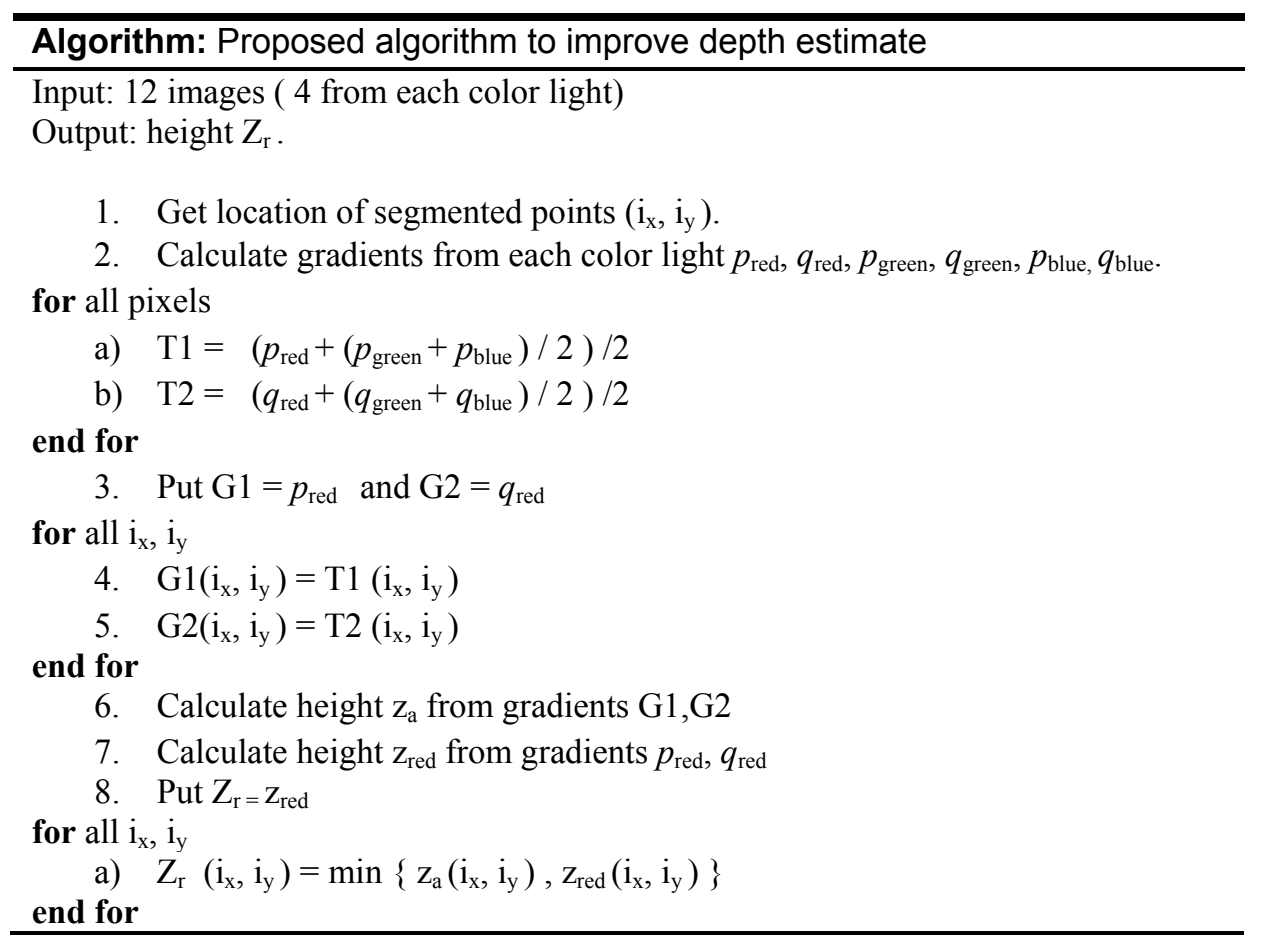


The algorithm minimized the errors when compared to white light and each R, G, B lights as shown in Table 1 and 2. Also the recovered shape of wrinkle from our algorithm counteracts the effects of any over and underestimation of wrinkle depth as shown in Fig. 5. The dotted line shows the ground truth from replica and the solid line profile is from the proposed algorithm. Overall the error was minimum for our algorithm when compared to all colour lights and subjects; however the accuracy of algorithm is currently dependent on the segmentation process.

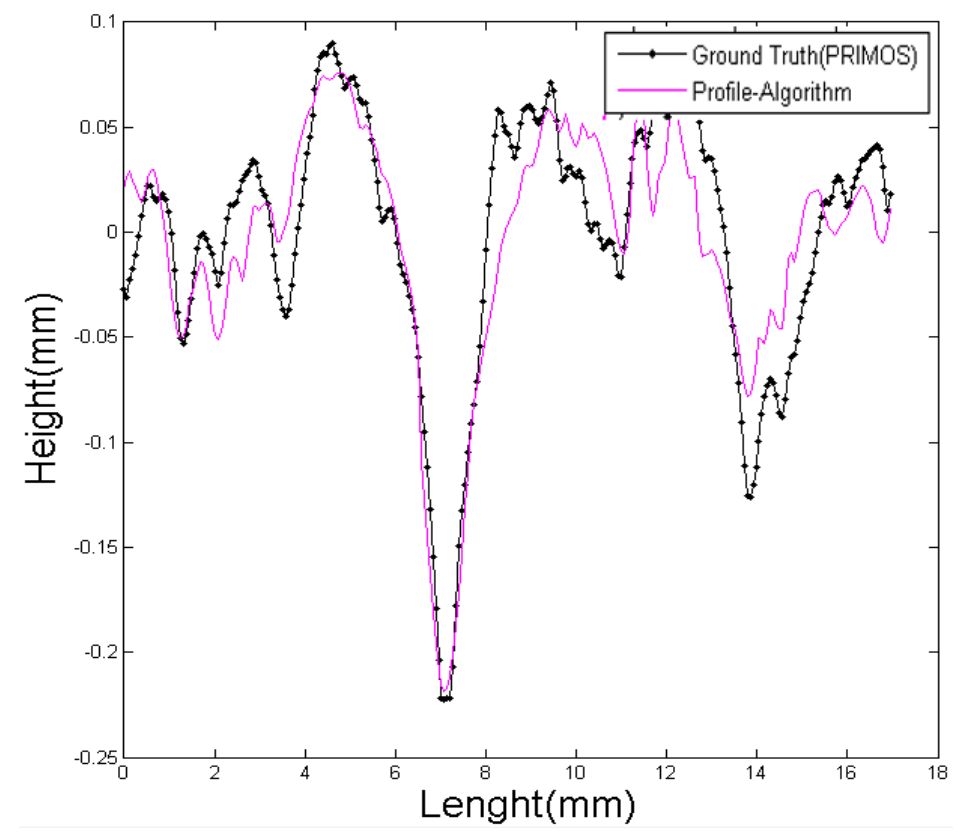

Fig. 5. Comparison of our algorithm with ground truth.

\section{Quantification Parameters}

The skin microrelief is anisotropic and changes with increasing age [27]. Most of the 3D imaging techniques to date have used the parameters defined by the metal industry for quantification of skin microrelief. The parameters used are defined in the ISO 468, ISO 4287 and DIN 4762-4768 for the characterization of metal surface [28]. The anisotropy and irregularity of skin relief makes characterization of skin different from metal that is why not all parameters are used for quantification of skin microrelief. It would be interesting to see how the surface and 3D aerial parameters defined in the ISO 25718 [29] help in characterization and quantification of skin relief. Especially the volumetric parameters: Peak material volume $\left(\mathrm{V}_{\mathrm{mp}}\right)$, Core material volume $\left(\mathrm{V}_{\mathrm{mc}}\right)$, Core void volume $\left(\mathrm{V}_{\mathrm{vc}}\right)$, Valley void volume $\left(\mathrm{V}_{\mathrm{vv}}\right)$.

The most commonly used parameters for roughness evaluation are $R_{a}, R_{z}, R_{p}, R_{t}$ [30]. These parameters have been used for analysis of age, effects of moisturizers, botox, sun damage, and have proved to be useful in characterization of before and after effects of treatments. Here we used three samples of forehead wrinkles representing low, moderate and high wrinkle states. Standard metal roughness parameters $R_{a}, R_{q}, R_{k u}, R_{s k}, R_{p}, R_{v}$ (Arithmetic mean, root mean square, kurtosis, skewness, largest profile peak height, largest profile valley depth respectively) along with Fractal box counting and power spectrum based techniques were used to quantify skin topography. Table 3 shows the quantitative comparison of three skin samples using conventional and fractal based technique. 
Table 3. Standard roughness and fractal based Quantification for different wrinkle states.

\begin{tabular}{|c|c|c|c|}
\hline & $\begin{array}{c}\text { Sample 1 } \\
\text { (low) }\end{array}$ & $\begin{array}{c}\text { Sample 2 } \\
\text { (moderate) }\end{array}$ & $\begin{array}{c}\text { Sample 3 } \\
\text { (high) }\end{array}$ \\
\hline $\mathrm{R}_{\mathrm{a}}$ & $10.4706 \mu \mathrm{m}$ & $47.1366 \mu \mathrm{m}$ & $54.2052 \mu \mathrm{m}$ \\
\hline $\mathrm{R}_{\mathrm{q}}$ & $12.3165 \mu \mathrm{m}$ & $57.3867 \mu \mathrm{m}$ & $65.6712 \mu \mathrm{m}$ \\
\hline $\mathrm{R}_{\mathrm{ku}}$ & $129.717 \mu \mathrm{m}$ & $144.1566 \mu \mathrm{m}$ & $154.1547 \mu \mathrm{m}$ \\
\hline $\mathrm{R}_{\mathrm{sk}}$ & $-4.1769 \mu \mathrm{m}$ & $17.3754 \mu \mathrm{m}$ & $-7.9632 \mu \mathrm{m}$ \\
\hline $\mathrm{R}_{\mathrm{p}}$ & $22.3146 \mu \mathrm{m}$ & $120.6135 \mu \mathrm{m}$ & $132.804 \mu \mathrm{m}$ \\
\hline $\mathrm{R}_{\mathrm{v}}$ & $24.5007 \mu \mathrm{m}$ & $100.4913 \mu \mathrm{m}$ & $123.0453 \mu \mathrm{m}$ \\
\hline $\begin{array}{c}\text { Fractal Dimension } \\
(\text { Box counting) }\end{array}$ & 2.0792 & 2.0911 & 2.1167 \\
\hline $\begin{array}{c}\text { Fractal Dimension } \\
\text { (Power spectrum) }\end{array}$ & 2.0573 & 2.0463 & 2.0995 \\
\hline
\end{tabular}

Unlike the conventional parameters for roughness analysis the fractal based technique does not require any high/ low pass filtering, and is independent of the anisotropy of skin ( the orientation of surface does not affect the quantification) and makes use of complete 3D skin topography. Fractal dimension is a non-integer dimension between the Euclidean dimension and it depends on the complexity of the surface. Fractal dimension for profiles exit between $1<D_{f}<2$ and for surfaces it lies between $2<D_{f}<3$, it is equal to Euclidean dimension of 1 for a straight line, 2 for a flat plane and 3 for a cube.

The power spectrum based fractal calculation was done using the Gwyddion software [31]. In the cube counting method for calculating fractal dimension, cubes of size (s) are counted which cover the whole surface. The cube size is then varied and the number of interesting cubes $N$ is counted. The log of cube count $\log (N(s))$ is then plotted against $\log (1 / s)$ and the slope of the resulting plot gives the required fractal dimension. Fig 6 (a)-(c) shows the variation of cube size in the cube mesh as it covers the whole surface. A comparison of skin roughness analysis using the conventional parameters and Fractal based technique shows that the resulting fractal dimension can be used in conjunction with $2 \mathrm{~d}$ roughness parameters for quantitative evaluation different wrinkle severities.

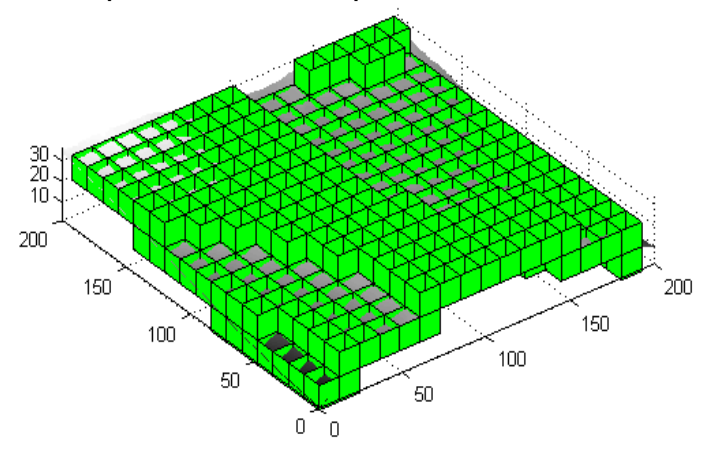

(a)

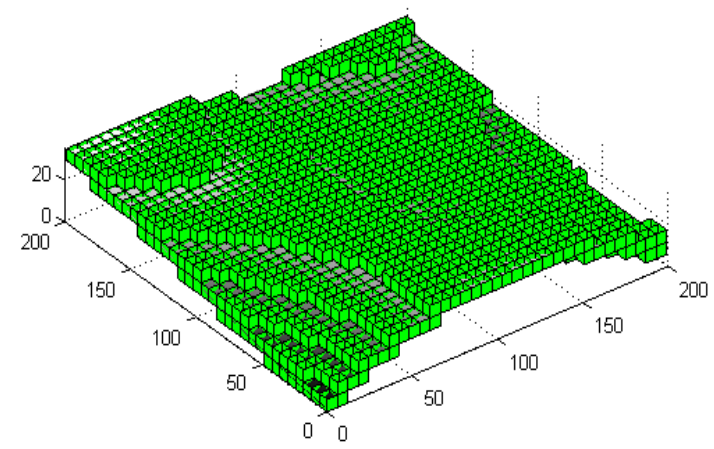

(b)

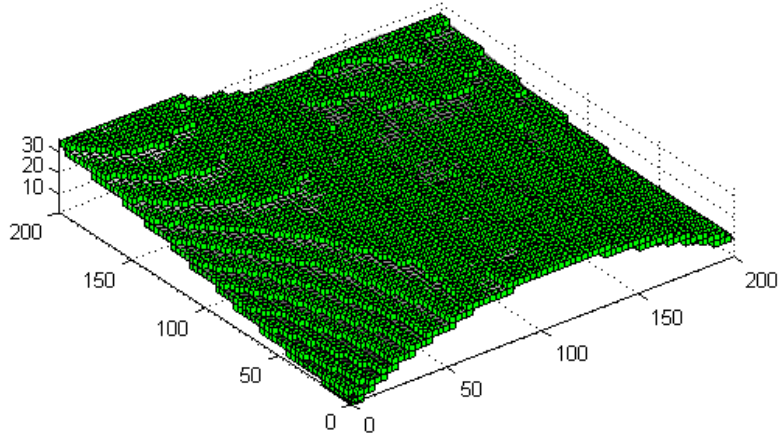

(c)

Fig. 6. Graphical representation of cube size variation ((a) - (c)) used for the calculation of Fractal dimension for a patch of skin. 


\section{Conclusion}

Current classification systems for wrinkles and microrelief in general are inadequate as a result of their qualitative nature and inherent problems with inter-user reproducibility. 3D in vivo profilometry provides objective analysis of skin microrelief and enables clinicians to predict and analyse the limitations and efficacy of various aesthetic procedures. The last decade has seen a shift toward 3D non invasive and in vivo measurements of skin roughness, however whilst numerous $3 \mathrm{D}$ techniques have been developed and used, none of them has been adopted as a standard. We have proposed a photometric stereo based technique for quantitative assessment of skin topography. Compared to other 3D imaging techniques it is economical, provides fast acquisition for everyday clinical usage and can easily recover skin colour and topographical information.

A new algorithm is proposed for interreflection removal in skin with varying reflectance and BRDF. The algorithm uses spectral and BRDF variation to overcome interreflections and has proven to work with both light and dark skin subjects. Fractals and conventional 2D profile analysis were also performed on different wrinkle states for quantitative assessment. The fractal based technique provided an added advantage over $2 \mathrm{D}$ roughness measurements by making use of complete $3 \mathrm{D}$ surface information and also did not require any high or low pass filtering.

\section{References}

1. K. Tsukahara, K. Sugata, O. Osanai, A. Ohuchi, Y. Miyauchi, M. Takizawa, M. Hotta, and T. Kitahara, (2007): "Comparison of age-related changes in facial wrinkles and sagging in the skin of Japanese, Chinese and Thai women", Journal of dermatological science, Vol. 47, No. 1, pp. 19-28.

2. G. D. Monheit, E. C. Gendler, B. Poff, L. Fleming, N. Bachtell, E. Garcia, and D. Burkholder, (2010): "Development and validation of a 6-point grading scale in patients undergoing correction of nasolabial folds with a collagen implant", Dermatologic surgery, Vol. 36, pp. 1809-1816.

3. A. Carruthers, J. Carruthers, B. Hardas, M. Kaur, R. Goertelmeyer, D. Jones, B. Rzany, J. Cohen, M. Kerscher, T. C. Flynn, C. Maas, G. Sattler, A. Gebauer, R. Pooth, K. McClure, U. Simone-Korbel, and L. Buchner, (2008): "A validated lip fullness grading scale", Dermatologic Surgery, vol. 34, pp. S161-S166.

4. A. Carruthers, J. Carruthers, B. Hardas, M. Kaur, R. Goertelmeyer, D. Jones, B. Rzany, J. Cohen, M. Kerscher, T. C. Flynn, C. Maas, G. Sattler, A. Gebauer, R. Pooth, K. McClure, U. Simone-Korbel, and L. Buchner, (2008): "A validated grading scale for marionette lines", Dermatologic surgery, vol. 34 Suppl 2, pp. S167-S172.

5. A. Carruthers, J. Carruthers, B. Hardas, M. Kaur, R. Goertelmeyer, D. Jones, B. Rzany, J. Cohen, M. Kerscher, T. C. Flynn, C. Maas, G. Sattler, A. Gebauer, R. Pooth, K. McClure, U. Simone-Korbel, and L. Buchner, (2008): "A validated grading scale for forehead lines", American Society for Dermatologic Surgery, vol. 34, pp. S155-S160.

6. G. Lemperle, R. E. Holmes, S. R. Cohen, and S. M. Lemperle, (2001): "A classification of facial wrinkles", Plastic and reconstructive surgery, vol. 108, no. 6, pp. 1751-1752.

7. C. Larnier, J. P. Ortonne, A. Venot, B. Faivre, J. C. Béani, P. Thomas, T. C. Brown, and E. Sendagorta, (1994): "Evaluation of cutaneous photodamage using a photographic scale", The British journal of dermatology, vol. 130, no. 2, pp. 167-73.

8. K. De Paepe, J. M. Lagarde, Y. Gall, D. Roseeuw, and V. Rogiers, (2000): "Microrelief of the skin using a light transmission method", Archives of dermatological research, vol. 292, no. 10, pp. 500-10.

9. S. Jaspers, (1999): "Rapid in vivo measurement of the topography of human skin by active image triangulation using a digital micromirror device", Skin Research and Technology, vol. 30, no. 3, pp. 129-207.

10. P. M. Friedman, G. R. Skover, G. Payonk, A. N. B. Kauvar, and R. G. Geronemus, (2002): "3D in-vivo optical skin imaging for topographical quantitative assessment of non-ablative laser technology", American Society for Dermatologic Surgery, vol. 28, no. 3, pp. 199-204.

11. D. Forsyth and A. Zisserman, (1989): "Mutual illumination", Proceedings CVPR: IEEE Computer Society Conference on Computer Vision and Pattern Recognition, pp. 466-473.

12. T. Yamada and H. Saito, (1998): " $3 d$ reconstruction of skin surface from image sequence", IAPR Workshop on Machine Vision, pp. 3-6.

13. A. Matsumoto, H. Saito, and S. Ozawa, (1999): "3D reconstruction of skin surface from photometric stereo images with specular reflection and interreflection", Electrical Engineering in Japan, vol. 129, no. 3, pp. 51-58. 
14. L. I. M. J. Vrhel, R. Gershon, (1994 ): "Measurement and analysis of object reflectance spectra", Color Research and Application, vol. 19, pp. 4-9.

15. K. J. Dana, (2001): "BRDF/BTF measurement device", ICCV, vol. 2, pp. 460-466.

16. K. J. Dana, B. Van Ginneken, S. K. Nayar, and J. J. Koenderink, (1999): "Reflectance and texture of real-world surfaces", ACM Transactions on Graphics, vol. 18, no. 1, pp. 1-34.

17. S. R. Marschner, S. H. Westin, E. P. F. Lafortune, K. E. Torrance, and D. P. Greenberg, (1999): "Image Based BRDF Measurement Including Human Skin", Eurographics Workshop on Rendering, pp. 131-144.

18. B. Koch, (2011): "A multispectral bidirectional reflectance distribution function study of human skin for improved dismount detection", PhD. Thesis, Airforce Institue of Technology.

19. J. Lagarde, C. Rouvrais, D. Black, S. Diridollou, and Y. Gall, (2001): "Skin topography measurement by interference fringe projection: a technical validation", Skin Research and Technology, vol. 7, no. 2, pp. 112-121.

20. R. Bazin and J. L. Lévêque, (2011): "Longitudinal study of skin aging: from microrelief to wrinkles", Skin research and technology, vol. 17, no. 2, pp. 135-40.

21. M. Setaro and A. Sparavigna, (2001): "Irregularity skin index (ISI): a tool to evaluate skin surface texture", Skin Research and Technology, vol. 7, no. 3, pp. 159-163.

22. T. Fujimura, K. Haketa, M. Hotta, and T. Kitahara, (2007): "Global and systematic demonstration for the practical usage of a direct in vivo measurement system to evaluate wrinkles", International journal of cosmetic science, vol. 29, no. 6, pp. 423-436.

23. E. T. Weiss, A. Chapas, L. Brightman, C. Hunzeker, E. K. Hale, J. K. Karen, L. Bernstein, and R. G. Geronemus, (2010): "Successful treatment of atrophic postoperative and traumatic scarring with carbon dioxide ablative fractional resurfacing: Quantitative volumetric scar improvement", Archives of dermatology, vol. 146, no. 2, p. 133.

24. M. C. T. Bloemen, M. S. van Gerven, M. B. a van der Wal, P. D. H. M. Verhaegen, and E. Middelkoop, (2011): "An objective device for measuring surface roughness of skin and scars", Journal of the American Academy of Dermatology, vol. 64, no. 4, pp. 706-15.

25. Y.H. Choi, Y.S. Tak, S. Rho, and E. Hwang, (2011): "Accurate Wrinkle Representation Scheme for Skin Age Estimation", Fifth FTRA International Conference on Multimedia and Ubiquitous Engineering, vol. 1, pp. 226-231.

26. B. Muralikrishnan, (2009): Computational surface and roundness metrology.

27. M. Setaro and A. Sparavigna, (2001): "Irregularity skin index (ISI): a tool to evaluate skin surface texture", Skin research and technology, vol. 7, no. 3, pp. 159-63.

28. M. Egawa, M. Oguri, T. Kuwahara, and M. Takahashi, (2002): "Effect of exposure of human skin to a dry environment", Skin Research and Technology, vol. 8, no. 4, pp. 212-218.

29. F. Blateyron, (2006): "New 3D parameters and filtration techniques for surface metrology", Japanese society of precision engineering annual congress.

30. T. H. Cook, (1980): "Profilometry of skin-a useful tool for the substantiation of cosmetic efficacy", J. Soc. Cosmet. Chem., vol. 31, no. December, pp. 339-359.

31. D. Nečas and P. Klapetek, (2011): "Gwyddion: an open-source software for SPM data analysis", Central European Journal of Physics, vol. 10, no. 1, pp. 181-188. 